\title{
Rechtsprobleme von privatisierter Sicherheit - Überlegungen zu einem verdrängten Phänomen
}

\section{Problemanfriss}

Die sog. »Publik Private Partnerships«, kommen sie als Sicherheits- oder Ordnungspartnerschaften, Sicherheitsnetzwerke oder sonstige öffentlich-private Sicherheitskooperationen ${ }^{\mathrm{I}}$ daher, befinden sich nicht nur im alltäglichen Blickfeld des aufmerksamen Bürgers, sondern auch unter Beobachtung der Polizeirechtswissenschaft ${ }^{2}$ sowie neuerdings - anlässlich einer entsprechenden Novelle im Jahre $2002^{3}$ - des Gewerberechts. ${ }^{4}$ Rechtsprobleme treten insbesondere bei der Frage der Kompetenzen von privaten Sicherheitsdienstleistern sowie anlässlich der unmittelbaren Kooperation von Polizei und Privaten - etwa bei gemeinsamen Streifengängen - zutage. Einen Unterfall der letztgenannten Problematik stellen Datenübermittlungen von Sicherheitsunternehmen an Sicherheitsbehörden - insbesondere die Polizei als Gefahrenabwehrbehörde einerseits sowie als Strafverfolgungsorgan andererseits - dar. ${ }^{5}$

Schließlich machen solche Sicherheitsfirmen von sich Reden, deren Anliegen darin besteht, in der Öffentlichkeit gerade nicht wahrgenommen zu werden. Gemeint sind insoweit - oft firmeninterne - Organisationen, die sich ausschließlich mit der Aufklärung von Straftaten innerhalb von Unternehmen befassen. Deren Ziel ist weniger, Straftäter einem »regulären« Strafverfahren zuzuführen, sondern vielmehr entstandene Schäden wieder gut zu machen und kriminelle Arbeitnehmer insbesondere schnell und ohne kosten- und zeitintensive Verfahren aus dem Unternehmen zu entfernen. ${ }^{6}$

I Vgl. dazu das Stichwort »Sicherheitspartnerschaften«, in: Martin H. W. Möllers (Hrsg.), Wörterbuch der Polizei, München 200I, I 459 sowie allg. Jörg Ziercke, Kooperationsfelder Polizei - Private Sicherheit, Die Polizei 95 (2004), 33 I-332.

2 Vgl. aus der Lehrbuchliteratur etwa Christoph Gusy, Polizeirecht, 6. Aufl. Tübingen 2006, 73 ff.; FranzLudwig Knemeyer, Polizei- und Ordnungsrecht, I I. Aufl. München 2007, I4 ff.; Dieter Kugelmann, Polizei- und Ordnungsrecht, Berlin 2006, I75 ff.; Hans Lisken/Erhard Denninger, Die Polizei im Verfassungsgefüge, in: dies. (Hrsg.), Handbuch des Polizeirechts, 4. Aufl. München 2007, I 35 ff.; Bodo Pieroth/ Bernhard Schlink/Michael Kniesel, Polizei- und Ordnungsrecht, 4. Aufl. München 2007, Ios ff.; WolfRüdiger Schenke, Polizei- und Ordnungsrecht, 5. Aufl. Heidelberg 2007, 280 ff.; Thomas Würtenberger/ Dirk Heckmann, Polizeirecht in Baden-Württemberg, 6. Aufl. Heidelberg 2005, I6 ff. sowie Martin Kutscha, Innere Sicherheit und Verfassung, in: Fredrik Roggan/Martin Kutscha (Hrsg.), Handbuch zum Recht der Inneren Sicherheit, 2. Aufl. Berlin 2006, 88 ff., jeweils m. zahlr. w. N.

3 BGBl. I 2002, $2724 \mathrm{ff}$.

4 Vgl. BT-Drucks. I4/8386 sowie die Anmerkungen von Anja Nünke/Claudia Deutschland, Kooperative Sicherheitsverantwortung in Staat und Gesellschaft, Die Polizei 95 (2004), $324 \mathrm{ff}$.

5 So wurde beispielsweise in Berlin die Feststellung von Parkverstößen einem Unternehmen übertragen, das die ermittelten Sachverhalte sodann der Polizei zur weiteren Bearbeitung weiterleitete. Diese Praxis wurde schließlich vom AG Tiergarten, NStZ-RR I3 (1996), 277 verworfen, weil Private nicht befugt seien, die von ihnen festgestellten Ordnungswidrigkeiten ohne Beteiligung eines Polizeibeamten »auszuermitteln «; insoweit zustimmend Rolf Stober, Staatliches Gewaltmonopol und privates Sicherheitsgewerbe, NJW 50 (1997), 889-896, 895; Gerhard Nitz, Privatisierung der öffentlichen Sicherheit Rechtsfragen am Beispiel der Privatisierung von Aufgaben der Verkehrsüberwachung, in: Christoph Butterwegge/Martin Kutscha/Sabine Berghahn (Hrsg.), Herrschaft des Marktes - Abschied vom Staat?, Baden-Baden 1999, I46-I 59, I 52 und Martin Kutscha, Sicherheit ohne Staat - Staat ohne Sicherheit?, in: Friedrich-Ebert-Stiftung (Hrsg.), Moderner Staat im Dialog, Band IV, Berlin 2007, I 5-27, 2 I.

6 Vgl. dazu etwa Rolf Rainer Jaeger, Problematik privater Ermittlungsorganisationen in Unternehmen, Der Kriminalist 40 (2008), I9-24. 
Es existieren wenige Branchen - nicht nur - in Deutschland, die sich über derartige Zuwächse freuen dürfen:7 Innerhalb der letzten 50 Jahre stieg die Anzahl der Unternehmen im Sicherheitsgewerbe von 600 auf mehr als 3000. Hierbei stieg die Zahl der dort Beschäftigten von weniger als 30000 auf heute I 45000 . Letztgenannte Zahl ist auch deshalb bemerkenswert, weil es aktuell nur noch die doppelte Anzahl von Mitarbeitern der »öffentlichen Polizei« gibt. Angesichts dieses Umstandes verwundert es kaum, dass mancherorts vor allem private Wachschützer - gerade in quasi-öffentlichen Räumen - durch die Wahrnehmung von Sicherheitsdienstleistungen auffallen. ${ }^{8}$ Der »Bundesverband Deutscher Wach- und Sicherheitsunternehmen « (BDWS) erkennt in der Betätigung seiner Mitglieder denn auch einen »unverzichtbaren Beitrag für die Innere Sicherheit in Deutschland «.9

Tatsächlich hat sich das Gewerbe auch nach gesetzgeberischer Erkenntnis über seine »klassischen« Aufgabenfelder wie Werkschutz, Objektschutz, Geld- und Werttransporte hinaus entwickelt und neue Tätigkeitsfelder, insbesondere im besonders stark anwachsenden Sektor der Bestreifung des öffentlichen und quasi-öffentlichen Verkehrsraums (etwa Bahnhöfen etc.) erobert. ${ }^{\circ}{ }^{\circ}$ Die zunehmende wirtschaftliche Macht des Sicherheitsgewerbes zeigt sich auch an seinem rasant steigenden Umsatz: Während dieser Anfang der neunziger Jahre noch bei ca. 2 Milliarden Euro lag, verdoppelte er sich bis 2002 auf ca. 4 Milliarden Euro ${ }^{\text {I }}$ und erreichte im Jahr 2006 mit 4,3 Milliarden Euro einen neuen Höchststand. ${ }^{\mathrm{I} 2}$

\section{Gewerberechtliche Regelungsansätze}

Als einer der wenigen legislativen Versuche der rechtlichen Einhegung privater Sicherheitsproduktion ist $\ 34$ a der Gewerbeordnung $(\mathrm{GewO})$ anzusehen. Demnach steht die entsprechende Betätigung unter dem Vorbehalt behördlicher Erlaubnis, die ihrerseits mit Auflagen verbunden werden kann. Die Erlaubnis ist insbesondere zu versagen, wenn Anhaltspunkte für eine fehlende Zuverlässigkeit des Antragstellers bestehen oder er einen Nachweis, dass er über die für die Ausübung des Gewerbes notwendigen rechtlichen Vorschriften unterrichtet worden und mit diesen vertraut ist, nicht erbringt ( $\mathbb{3 4}$ a Abs. I GewO).

Über diesen auf die Einrichtung und Ausübung des Betriebes bezogenen Erlaubnisvorbehalt hinaus besteht für bestimmte Angestellte im Bewachungsgewerbe das Erfordernis einer abgelegten Sachkundeprüfung. Betroffen hiervon sind Personen, die Kontroll- bzw. Streifengänge im öffentlichen Verkehrsraum oder in »Hausrechtsbereichen mit tatsächlich öffentlichem Verkehr« wahrnehmen. Dasselbe gilt für Ladendetektive oder sog. »Türsteher « vor Diskotheken $(\mathbb{S}$ 34a Abs. I Satz 4 GewO). Diese Angehörigen privater Sicherheitsdienstleister müssen u.a. über (Grund-)Kenntnisse des Polizei- und Strafrechts, des Gewerberechts, bewachungsspezifische Aspekte des Datenschutzes sowie des Verhaltens in Gefahrensituationen und Deeskalationstechniken in Konfliktsituationen

7 Nachweise im Einzelnen bei Stephan Rixen, Vom Polizeirecht über das Gewerberecht zurück zum Polizeyrecht?, DVBl. I 22 (2007), 22 I-23 I, 223.

8 Vgl. etwa Christoph Gusy, Polizei und Sicherheitsdienste im öffentlichen Raum, VerwArch 92 (200I), $344-367,345$ f.; BT-Drucks. I4/8386, S. I I.

9 So die Selbstdarstellung des BDWS unter www.bdws.de.

Io BT-Drucks. I $4 / 8386$, S. I I.

I I Rixen (Fn. 7), 223.

I2 Quelle www.bdws.de. 
verfügen. ${ }^{13}$ Worüber diese Grundlagen nicht informieren können, sind freilich die gesetzgeberischen Auslassungen, denn beispielsweise das Polizeirecht kennt ja gerade keine Kooperationen mit nicht-polizeilichen »Streifengängern«. Unabhängig hiervon verwundert es, dass der Gesetzgeber sich auf die Kodifizierung selbständiger Betätigung beschränkt hat und die Vorgaben des $\ 34 \mathrm{a}$ GewO damit keine Geltung für unselbständige Unternehmensbereiche (etwa der Deutschen Bahn AG) beanspruchen kann. ${ }^{14}$

\section{II. Über die Gefahr des Übergangs der Polizeigewalt auf Private}

\section{Zur potentiellen Demokratiegefährlichkeit privater Sicherheitsproduktion}

Einer der Anknüpfungspunkte für verfassungsrechtliche Bedenken besteht in der Besorgnis fehlender demokratischer Kontrolle privater Sicherheitskräfte: Wer die Verfügungsrechte über solche Kräfte besitzt, konkurriert mit dem Staat um die Innehabung der Entscheidungsmacht über den Einsatz organisierter Gewalt. Zwar ist die von Stümper beschriebene Gefahr einer »Umwidmung « dieses personellen Potentials zu einer zu politischen Zwecken nutzbaren »schlagkräftigen Einheit « nicht aktuell, immerhin aber durchaus nicht abwegig. Is

In der Tat erscheint es auf den ersten Blick - auch bei Berücksichtigung der inzwischen zum Normalfall gewordenen Wahrnehmung öffentlicher Sicherheitsaufgaben auf Bahnhöfen und anderen tatsächlich öffentlichen Orten durch »Security«-Leute - nicht akut, dass der moderne demokratische Verfassungsstaat beim sich weiterhin weitgehend unreglementiert entwickelnden privaten Sicherheitsgewerbe mit hinreichender Wahrscheinlichkeit durch eine demokratiegefährdende Struktur bedroht würde. Indessen - und hier sei ein Ausflug in polizeirechtliche Terminologie gestattet: Auch eine abstrakte Gefahr, also eine Sachlage, bei der nach allgemeiner Erfahrung eine hinreichende Wahrscheinlichkeit besteht, dass ein zu schützendes Gut geschädigt wird, ermächtigt zum staatlichen Eingreifen. Und: Je größer der drohende Schaden ist, desto geringer sind die Anforderungen an die Eintrittswahrscheinlichkeit. ${ }^{16}$ Aus diesem Blickwinkel betrachtet, stellen private Uniformierte in Bahnhöfen und Einkaufspassagen zwar keine konkrete Bedrohung des jedenfalls aktuell nicht instabilen demokratischen status quo dar. Wohl aber erscheint es durchaus nicht abwegig, dass eine weitgehende legislative Untätigkeit unter demokratischen Gesichtspunkten in verstärktem Maße rechtfertigungsbedürftig erscheint. ${ }^{17}$

\section{Sicherbeit als marktgängige Ware}

Ein weiterer Anlass für eine kritische Begleitung der Übernahme von Sicherheitsaufgaben durch Private ist ein rechtlich und tatsächlich nur schwer kontrollierbarer »Angstmarkt«. Kriminalitätsfurcht und allgemeines Unsicherheitsgefühl, das gerade auch in manch populistisch geführtem Wahlkampf eine Rolle spielt, geht mit dem »Verwertungsinteresse « von Sicherheitsgewerbe und Sicherheitsindustrie eine durchaus unheilige Allianz ein: Aus dem Blickwinkel einer Vorsorge für den

I 3 Vgl. zu den Sachgebieten der Sachkundeprüfung sowie den Stoffkatalog www.ihk-nordwestfalen.de.

I 4 Krit. dazu Andreas Peilert, zitiert nach Nünke/Deutschland (Fn. 4), 328.

is Wolfgang Hoffmann-Riem, ZRP io (1977), 277-284, 279.

I6 Vgl. dazu etwa Erhard Denninger, Polizeiaufgaben, in: Hans Lisken/Erhard Denninger (Hrsg.), Handbuch des Polizeirechts, 4. Aufl. München 2007, 322.

I7 Vgl. auch Lisken/Denninger (Fn. 2), I 36 ff. 
zukünftigen Erfolg am Sicherheitsmarkt muss nämlich ein Gefühl der privaten Verantwortung für die eigene Sicherheit gefördert werden. Dieses Gefühl - so etwa Hoffmann-Riem - ist aber nur so lange unbedenklich, wie der Einzelne nicht meint, den Bereich seiner Verantwortung so ausdehnen zu sollen, dass er sich außerhalb des persönlichen Einwirkungsbereichs für die Erhaltung der öffentlichen Sicherheit oder gar die Strafverfolgung verantwortlich wähnt. ${ }^{18}$

Wenngleich die Verortung dieser (gesellschafts-)politischen Kosten im Verfassungsrecht durchaus nicht selbstverständlich erscheint, so sind diese Befürchtungen doch von mittelbarer Relevanz für die innergesellschaftliche Freiheit: Tatsächlich ist die Ausdehnung privater Sicherheitsvorsorge in den öffentlichen Bereich hinein nicht nur ein vielfältig zu beobachtender Befund, sondern war auch bereits wiederholt Gegenstand judikativer Befassung, auf die im Folgenden noch einzugehen sein wird. ${ }^{19}$ Namentlich bei vorsorglichen Datenerhebungen, insbesondere Videoüberwachungen, scheinen Private zur Vereinnahmung der Öffentlichkeit zu neigen. Einen bemerkenswerten Höhepunkt fand dieses Phänomen in der Behauptung eines Berliner Kaufhaus-Eigners, sich für die Terrorismusbekämpfung auf dem vorgelagerten (tatsächlich öffentlichen) Straßenraum zuständig zu fühlen. ${ }^{20}$

Jenseits solcher Kompetenzüberschreitung (und -überschätzung) führt öffentliche Sicherheit als marktgängige Ware aber jedenfalls auch dazu, dass eben jene Mechanismen wirken, die typischerweise bei wirtschaftlicher Betätigung virulent sind. ${ }^{21}$ Hierzu zählt namentlich die angesprochene Vorsorge für zukünftige Chancen zur Gewinnerzielung und damit im Fall der privaten Sicherheitsdienstleister das ggf. zu »provozierende« Verlangen nach Sicherheitsproduktion. Insoweit liegt es durchaus in staatlicher Verantwortung, den so bezeichneten »Angstmarkt « durch eine rationale Sicherheitspolitik zu zähmen. Hiermit ist jedenfalls das trivialisierende Thematisieren von Kriminalitätsentwicklungen unvereinbar. ${ }^{22}$

\section{Verdrängung staatlicher Rechtsgutssicherung}

Als weiterer, durchaus mit Verfassungsrang ausgestatteter Grundsatz, der mit der Ausweitung privater Sicherheitsdienstleistungen im strukturellen Konflikt steht bzw. stehen kann, ist das Gewaltmonopol, also das vorrangige Recht zur Sicherung individueller und allgemeiner Rechtsgüter. Hervorzuheben ist insoweit, dass es als rechtsstaatliche Errungenschaft anzusehen ist, dass strafrechtliche Privatjustiz weitreichend durch die bei ihren Eingriffen auf gesetzlich bestimmte Handlungsformen und Instrumente beschränkte Staatsgewalt im modernen Verfassungsstaat ersetzt worden ist. Die damit zu bewältigende Problematik liegt darin, dass private Gewalt sehr viel schwerer kontrollierbar ist, als das bei den vielfältig kontrollierbaren staatlichen Instanzen der Fall ist. ${ }^{23}$

Ein durchaus herausstechendes Beispiel ist die einleitend beschriebene Betriebspolizei- und -justiz, die Einzelfallgerechtigkeit und Verfahrensgarantien außer Betracht lassen und sich auf eine am Effektivitätsgrundsatz einschließlich Wirtschaftlichkeitskriterien orientierte Konfliktbewältigung beschränken kann. Hier zeigt sich, dass die privat angeeignete Erledigung von Delinquenz zur Ausschal-

I 8 Hoffmann-Riem (Fn. I5), 279.

I9 $B G H$, NJW 48 (I995), I955 sowie das unter III. angeführte Beispiel.

20 So das Prozessvorbringen im unter III. angeführten Beispiel, vgl. dazu die Presseerklärung der Humanistischen Union v. 20.1 I.2002 unter www.humanistische-union.de.

2I Vgl. dazu auch Martin Kutscha, NJ 5 I (1997), 393-397, 394.

22 So aber zuletzt geschehen im Hessischen Wahlkampf des Jahres 2008, krit. dazu etwa Heribert Prantl, Süddeutsche Zeitung v. 9.I.2008.

23 Hoffmann-Riem (Fn. I 5), 279. 
tung der staatlichen Justizorgane führen kann, die ihrerseits mit einer nicht unerheblichen Schlechterstellung von Betroffenen einhergeht, weil - in dieser Erscheinungsform - arbeitsrechtliche Garantien schlicht leer laufen.

\section{Der Sozialstaatsgrundsatz als Schutz vor privater Privilegiensicherung}

Schließlich ist es privater Sicherheitsproduktion eigen, dass sie sich am individuellen Interesse des Auftraggebers orientiert. Es kann den beauftragten Firmen daher beispielsweise genügen, wenn sie zur Verdrängung bestimmter Erscheinungsformen von Kriminalität in andere Bereiche beitragen. Solche Verdrängungseffekte ihrerseits können durchaus, wie sich an der Verarmung und damit einhergehender, steigender Kriminalitätsbelastung ganzer Stadtviertel - insbesondere in den Großstädten - in bedrückender Weise zeigt, zu einer »Eskalation der Intensität und Gefährlichkeit « von Delinquenz führen. ${ }^{24}$

Mit dem Gebot eines »Ausgleichs der sozialen Gegensätze«, wie es das Bundesverfassungsgericht als Ausprägung des Sozialstaatsprinzips versteht, ${ }^{25}$ ist eine solche Strategie schwerlich in Einklang zu bringen. Indessen handelt es sich bei ihr um eine unvermeidbare Auswirkung, wenn öffentliche Sicherheit zur marktgängigen Ware wird und damit vor allem denjenigen zur Verfügung steht, die die erforderlichen Ressourcen aufzubringen in der Lage sind. Freilich ist ebenso wenig zu verkennen, dass auch Jedermann innerhalb von in privatwirtschaftlicher Verantwortung geschützten Bereichen von der entsprechenden Sicherheitsproduktion zu profitieren vermag.

\section{Gesetzliche Zähmung privater Sicherheitsproduktion?}

Insgesamt stellt sich die Reglementierung der privaten Sicherheitsproduktion als bemerkenswert fragmentarisch dar: Als beinahe zufällige Einsprengsel finden sich an höchst unterschiedlicher Stelle Normen, die ihrerseits der kritischen Würdigung bedürfen. Im Rahmen dieses Beitrages können nur zwei besonders »prominente« Problemfelder behandelt werden. Dagegen müssen andere Rechts-Phänomene, ${ }^{26}$ wie etwa Datenübermittlungen zwischen privaten Sicherheitsdiensten und staatlichen Sicherheitsbehörden, ${ }^{27}$ hier weitgehend außer Betracht bleiben. Wie wenig wirkungsvoll die vorhandenen Regelungsansätze sind, soll an zwei Problemfeldern illustriert werden.

\section{I. "Zwangsmittel « für private Security?}

Das bislang noch geltende Gewerberecht ${ }^{28}$ geht - ohne dass dies eine nähere Begründung erführe - davon aus, dass Sicherheitsfirmen und deren Angehörige bei der Durchführung von Bewachungsaufgaben gegenüber Dritten nur die Rechte ausüben dürfen, die Jedermann im Falle einer Notwehr, eines Notstandes oder einer Selbsthilfe sowie etwaig übertragener Selbsthilferechte zustehen. Bei der Inanspruchnahme dieser Rechte ist lediglich der Grundsatz der Erforderlichkeit zu beachten (vgl. \$ $34 \mathrm{a}$ Abs. s GewO). Gerade letztgenannte Restriktion ist

\footnotetext{
24 Hoffmann-Riem (Fn. I5), 280.

25 BVerfGE 100, 27I (284).

26 Hierzu zählt etwa der Waffeneinsatz durch private Sicherheitsdienstleister einschließlich des Einsatzes von Hunden.

27 Vgl. hierzu nun das novellierte Berliner Datenschutzgesetz ( $\$$ 3 rb Abs. 3a BlnDSG - Videoüberwachung). 28 Vgl. hierzu Art. I25a GG (Weitergeltung von Bundesrecht nach Föderalismusreform).
} 
offensichtlich dem allgemeinen Verhältnismäßigkeitsgrundsatz entlehnt, der im

Bereich der öffentlichen Polizeigewalt einen omnipräsenten Rechtmäßigkeitsmaßstab darstellt. Als »zweite Stufe« einer jeden Verhältnismäßigkeitsprüfung fordert er - ohne dass dies hier einer näheren Betrachtung bedürfte - den Einsatz des »mildesten Mittels«. Im Umkehrschluss gilt dann aber, dass privaten Sicherheitsdienstleistern die Prüfung der Zumutbarkeit bzw. Angemessenheit als dritter Stufe der Verhältnismäßigkeit nicht abverlangt wird. ${ }^{29}$ Eine solche Privilegierung privater Sicherheitsdienstleister ist nicht einsichtig und führt gerade in Fällen, in denen sog. »gemischte Streifen « in tatsächlich öffentlichen Bereichen ihren Dienst tun, ${ }^{3 \circ} \mathrm{zu}$ dem wenig überzeugenden Ergebnis, dass von privater Security auch solche Maßnahmen ergriffen werden dürfen, die ein Polizeibeamter wegen unangemessener Belastung eines Betroffenen nicht anordnen dürfte. Abgesehen davon, dass der Gesetzgeber hier einen Teilaspekt des Verhältnismäßigkeitsgrundsatzes auf private Sicherheitskräfte anwendet, diese damit staatlicher Polizei befugnismäßig annähert und hiermit möglicherweise ein schlechtes Gewissen ob seiner Untätigkeit im Übrigen offenbart, ${ }^{3 \mathrm{I}}$ besteht hier eine schwer erträgliche Widersprüchlichkeit de lege lata: Soll etwa der Angehörige der Bahn-Tochter »Station \& Service« den friedlichen, aber bereits mit einem Hausverbot belegten Betrunkenen ohne Reiseabsichten des frostigen Winters tatsächlich des Bahnhofs(platzes) verweisen dürfen, während der daneben stehende Bundespolizist zwischen Bewunderung ob der "privaten Machtfülle« einerseits und dem Fertigen einer Strafanzeige wegen Aussetzung $(\mathbb{2} 2 \mathrm{I}$ StGB) gegen seinen »Streifenkollegen« andererseits schwankt?

Gegen eine solche Übertragung von »Jedermann-Rechten « auf private Sicherheitsdienstleister sprechen gewichtige Gründe: Bei Anerkennung des grundsätzlichen Vorrangs staatlichen Schutzes privater Rechte als polizeilicher Aufgabe32 ist die Berufung auf Notrechte nur in Ausnahmefällen statthaft. Denn den strafrechtlichen Normen über zulässigen Selbst- und Fremdschutz ist eigen, dass sie einen Bruch im System eines rechtsstaatlich gebändigten Ausgleichs von kollidierenden Individualinteressen bedingen. Nicht etwa gibt es ein Recht zur Selbsthilfe, Notwehr etc.; lediglich ausnahmsweise können entsprechende Eingriffe in die Rechte anderer als nicht rechtswidrig oder schuldhaft anzusehen sein. 33 »Professionelle Nothelfer« dürfen solche Ausnahmevorschriften dagegen nicht als Befugnisnormen in Anspruch nehmen, denn dann würden diese Regelungen ihrerseits eine neue Qualität erlangen, die mit ihrer Ausgestaltung aber unvereinbar wäre. ${ }^{34}$ Es geht demzufolge um eine teleologische Reduktion, die die allgemeinen Notrechte nur in solchen Situationen greifen lässt, in denen das insoweit typische Überraschungsund Unterlegenheitsmoment besteht, das gerade aus fehlender Möglichkeit zur Vorbereitung auf gefahrenabwehrende Maßnahmen gekennzeichnet ist. 35

Tatsächlich entspricht es heute der wohl überwiegenden Meinung, dass insbesondere die allgemeinen Rechtfertigungsgründe keinen Befugnischarakter im Hinblick auf polizeiliches Eingriffshandeln haben. ${ }^{6}$ Wenn und solange dem so ist, können diese Vorschriften jedenfalls dann auch nicht für die Tätigkeit von professionellen

29 Krit. hierzu Lisken/Denninger (Fn. 2), I 37 f.

30 Vgl. zur entsprechenden Praxis Thomas Brunst/Jürgen Korell, Private Sicherheitsunternehmen und Polizei, unter www.safercity.de/200I/privat.html, dort unter »Kooperation mit der Polizei«.

3I Rixen (Fn. 7), 227.

32 Hoffmann-Riem (Fn. I 5 ), 280.

33 Pieroth/Schlink/Kniesel (Fn. 2), ı०8.

34 A. A. Stober (Fn. 5), 893 ff.

35 Ebenso Rixen (Fn. 7), 225 und Kutscha (Fn. 5), 24.

36 Pieroth/Schlink/Kniesel (Fn. 2), 2 I9 f.; Schenke (Fn. 2), I8 u. 320; Gusy (Fn. 2), 84 f; Würtenberger/ Heckmann (Fn. 2), 374; Kutscha (Fn. 2), 42. 
Sicherheitsdienstleistern herangezogen werden, wenn diese zum Schutz von öffentlichrechtlich geschützten Rechtsgütern der Allgemeinheit tätig werden. ${ }^{37}$ Vielmehr bedürfte es nach richtiger Ansicht einer gesetzgeberischen Festlegung, welche Befugnisse private Sicherheitsdienstleister unter welchen Voraussetzungen in Anspruch nehmen dürfen. $3^{8}$ Dies hat erhebliche Folgen für die Sicherheits-Wirklichkeit auf Bahnhöfen, Flughäfen und anderen tatsächlich öffentlichen Flächen: Da insoweit ein Verstoß gegen den Vorbehalt des Gesetzes zu konstatieren ist, stellt sich die Tolerierung der Übernahme öffentlicher Sicherheitsaufgaben durch den Staat als verfassungsrechtlich unzulänglich dar. Nur auf diese Weise kann auch den Grundrechten in privatisierten öffentlichen Räumen39 angemessen Geltung verschafft werden. Es geht damit um nicht weniger als - wie Kersten und Meinel treffend beschreiben - die normative Rekonstruktion von Öffentlichkeit. ${ }^{\circ}$ Kernstück einer solchen Rekonstruktion hätte insbesondere die Reglementierung der einsetzbaren Zwangsmittel durch privatisierte Security, einschließlich der umfassenden Geltung des Verhältnismäßigkeitsgrundsatzes zu sein.

In der Praxis lassen sich auch die eigentlich zu trennenden Aufgabenbereiche von Mitarbeitern des privaten Sicherheitsgewerbes einerseits und Polizeibeamten andererseits »auf der Straße« (Pitschas) nicht trennscharf unterscheiden, so dass die an sich sowohl optisch wie auch akustisch erforderliche Abschottung bei der jeweiligen Aufgabenwahrnehmung - etwa bei Personenkontrollen bzw. Identitätsfeststellungen - bislang nicht gewährleistet ist. ${ }^{4}$ Immerhin müsste der private Sicherheitsdienstleister ja, wenn er aus bestimmten Gründen ein Interesse an der Kenntnis der Personalien einer Person hat, auf die Freiwilligkeit der entsprechenden Angaben hinweisen oder - befindet er sich in Begleitung eines zur Identitätsfeststellung befugten Polizeibeamten - »weghören «, während dieser eine ggf. mit Zwangsmitteln durchsetzbare ${ }^{42}$ - und regelmäßig mit Folgeeingriffen verbundene - Identitätsfeststellung43 trifft.

Freilich bliebe es auch bei entsprechenden legislativen Bemühungen bei einer gefährlichen Infragestellung eines Verfassungsprinzips:44 Denn Art. 33 Abs. 4 GG statuiert mit dem Funktionsvorbehalt eine Pflicht für die Gesetzgeber, öffentliche Gewalt grundsätzlich Beamten vorzubehalten4s und damit private Beliehene allenfalls ausnahmsweise mit der Wahrnehmung öffentlicher Sicherheitsaufgaben zu betrauen. ${ }^{6}$ Eine ständige Übertragung hoheitlicher Befugnisse in größerem Umfang ist daher - auch nach der Rechtsprechung des Bundesverfassungsgerichts 47 - verfassungswidrig. Unter diesem Blickwinkel sind selbst Regelungen, die die Begrenzung privater Befugnisse bei der Wahrnehmung öffentlicher Sicherheitsaufgaben zum Gegenstand haben (vgl. etwa den unter

37 Gusy (Fn. 8), 355; Schenke (Fn. 2), 282.

38 Für ein »Gesetz über Aufgaben und Befugnisse des Sicherheitsgewerbes « Rainer Pitschas, «Sicherheitspartnerschaften « der Polizei und Datenschutz, DVBl. IIs (2000), I805-18I5, I806; Pieroth/Schlink/ Kniesel (Fn. 2), I08; Schenke (Fn. 2), 282.

39 Zur Funktion des öffentlichen Raums ausführlich Gusy (Fn. 8), 348 ff.

40 Jens Kersten/Florian Meinel, JZ 62 (2007), I I 27 -I I 34, I I 34.

4I Rixen (Fn. 7), 226; Pitschas (Fn. 38), I8 I3.

42 Vgl. zur Durchsetzung der Maßnahme nur Pieroth/Schlink/Kniesel (Fn. 2), $249 \mathrm{f}$.

43 Vgl. hierzu nur Frederik Rachor, Das Polizeihandeln, in: Lisken, Hans/Denninger, Erhard (Hrsg.), Handbuch des Polizeirechts, 4. Aufl. München 2007, 528

44 Zur Begründung dieses Grundsatzes jüngst Gerhard Hornmann, Zur Unvereinbarkeit des Hessischen Freiwilligen-Polizeidienst-Gesetzes mit Art. 33 Abs. 4, LKRZ 2 (2008), 20I-206, 20 I f.

45 Vgl. dazu nur die Nachweise bei Hans D. Jarass/Bodo Pieroth, Grundgesetz für die Bundesrepublik Deutschland, 8. Aufl. München 2006, Art. 33 Rn. 40 ff.

46 Ausführlich hierzu Christof Gramm, Schranken der Personalprivatisierung bei der inneren Sicherheit, VerwArch 90 (1999), 329-360; vgl. auch Gusy (Fn. 8), 355 ff.

47 BVerfGE 9, 268 (284). 
Bestimmtheitsgesichtspunkten verunglückten $\ 5$ Abs. s Luftsicherheitsgesetz), ${ }^{48}$ als kompetenzüberschreitend aufzufassen. ${ }^{49}$ Es zeigt sich, dass sich Zwangsbefugnisse in den Händen von Privaten angesichts der geltenden Verfassungslage in den genannten Konstellationen als grundsätzlich zweifelhaft darstellen. ${ }^{\circ}$

\section{Entgrenzte Videoüberwachungen im öffentlichen und quasi-öffentlichen Raum}

Als weitere Ausprägung der Aneignung des öffentlichen und quasi-öffentlichen Raums sind - in ihrer Effizienz im Einzelfall durchaus anzuzweifelndes ${ }^{\mathrm{I}}-$ Videoüberwachungen durch Private anzusehen.52 Als einzige Restriktion findet sich insoweit eine Regelung im Bundesdatenschutzgesetz ( $\int 6 \mathrm{~b}$ BDSG), die nach ihrem Wortlaut sämtliche öffentlich zugänglichen Räume meint.53 Solcherlei Videoüberwachung ist erlaubt - soweit sie von nicht-öffentlichen Stellen durchgeführt wird - zur Wahrnehmung konkret festgelegter, berechtigter Interessen oder des Hausrechts. Begrenzt wird die Befugnis lediglich durch schutzwürdige Interessen der Betroffenen, wenn diese überwiegen ( $\ 6 \mathrm{~b}$ Abs. I BDSG).

Erkennbar enthält die Vorschrift keine Restriktionen, wie sie etwa aus polizeirechtlichen Regelungen über die optische (und teilweise akustische) Kontrolle von öffentlichen Plätzen oder Objekten bekannt sind.54 Anlass zu notwendiger Kritik bietet sie schon deshalb, weil in vielen Fällen für die Betroffenen schon nicht erkennbar ist, wo das Hausrecht des Eigentümers beginnt und die öffentliche Sphäre endet.55 Auch ist die Frage einer Ungleichbehandlung von öffentlichen und nicht-öffentlichen Bereichen - etwa auf Bahnhöfen - nach wie vor nicht beantwortet, denn für den Bürger ist öffentliche und private Videoüberwachung kaum zu unterscheiden. Jeweils muss er in den überwachten Bereichen mit der Erfassung seines Abbildes - gegebenenfalls auch in Nahaufnahme - rechnen. ${ }^{6}$ Die Vorschrift verschwindet gar im semantischen Niemandsland, soweit sie die Videoüberwachung zur Wahrnehmung berechtigter Interessen für festgelegte Ziele gestattet. $\mathrm{Ob}$ insofern die Forderung, wonach die zu beachtenden schutzwürdigen Belange der Betroffenen eine verfassungskonforme Auslegung dergestalt verlangen, dass lediglich die »Gewährleistung von Sicherheit« die Maßnahmen rechtfertigen können, ist angesichts der Wirklichkeit der Videoüberwachung nachhaltig zu bezweifeln. Vielmehr lässt der real existierende $\mathbb{6} 6$ BDSG doch eher den Schluss zu, dass privaten Interessen, die bis zum gezielteren Einsatz von Werbemitteln durch die Ermittlung der tatsächlichen Nutzung von bestimmten Bereichen zu bestimmten Tageszeiten reichen kann, der Vorrang vor dem Anspruch der Bürger auf unbeobachtete Nutzung tatsächlich öffentlicher Flächen eingeräumt wurde. Andernfalls hätte eine unter dem Gesichtspunkt der

48 Allein auf dem Flughafen von Frankfurt sollen 30 private Sicherheitsunternehmen mit I 900 Beschäftigten tätig sein, vgl. Nünke/Deutschland (Fn. 4), 328.

49 Schenke (Fn. 2), 282; Kutscha (Fn. 22), 394; Pitschas (Fn. 38), I8 16; a.A. Rupert Scholz, Verkehrsüberwachung durch Private?, NJW 50 (I997), I4-18, I 5: »Teilhabe« der Privaten am staatlichen Gewaltmonopol; vgl. auch Andreas Peilert, Police Private Partnership, DVBl. I I4 (I999), 282-29I, 285.

s० Kutscha (Fn. 2), 90; ders. (Fn. 5), 2 I ff.

5 I Vgl. zur Evaluation der Videokontrolle durch das Berliner Nahverkehrsunternehmen BVG krit. die Humanistische Union unter http://berlin.humanistische-union.de/themen/videoueberwachung.

52 Grds. krit. hierzu Jan Wehrheim, Ein fast ignoriertes Überwachungsdrama, Forum Wissenschaft 2/2000, $34-40$.

53 Näher hierzu Peter Gola/Rudolph Schomerus, Bundesdatenschutzgesetz, 9. Aufl. München 2007, \6b Rn. 8 f.; vgl. auch Thomas Königshofen, RDV I7 (200I), 220-223, 220.

54 Ausführlich dazu Fredrik Roggan, Polizeiliche Videoüberwachungen von öffentlichen Plätzen, in: Fredrik Roggan/Martin Kutscha (Hrsg.), Handbuch zum Recht der Inneren Sicherheit, 2. Aufl. Berlin 2006, 207-227 m. w. N.

55 Thilo Weichert, Rechtsfragen der Videoüberwachung, DuD 25 (2000), 662-669, 666.

56 Roggan/Kutscha (Fn. 54), 2 I 2. 
Normenklarheit wesentlich näher liegende Beschränkung auf die Sicherheitsgewährleistung vorgenommen werden müssen. 57

Diese Mängel finden ihre Bestätigung in der bislang vereinzelten Rechtsprechung zur Zulässigkeit privater Überwachung privat überbauten Straßenlandes, das durch Widmung zur Benutzung durch die Öffentlichkeit bestimmt ist. Das Amtsgericht Berlin-Mitte etwa hat in einem ersten Fall nur unter erheblichem Begründungsaufwand feststellen mögen, dass die Videokontrolle nur jenseits eines einen Meter breiten Streifens ab der Hauswand eine Verletzung der schutzwürdigen Belange der Passanten darstellt. ${ }^{8}$ Wer mithin die gesamte Breite des entsprechenden Fußweges nutzt, muss, da die kameraüberwachten Bereiche selbstverständlich nicht entsprechend markiert sind, mit seiner jederzeitigen Erfassung rechnen. Was sich im entschiedenen Einzelfall als erträglicher Ausgleich zwischen den Interessen des Klägers und denen des beklagten Kulturkaufhauses darstellen mag, offenbart aber doch das grundlegende Dilemma privater Überwachung öffentlicher Räume: Die letztlich kaum reglementierte Aneignung der Allmende durch Private und deren (Sicherheits-)Interessen.

\section{Käufliche Sicherheit als Herausforderung für den Gesetzgeber}

Als »janusköpfig « hat Kutscha den schlanken Staat, der ohne die Privatisierung von staatlichen Aufgaben nicht auszukommen scheint, in der Kritischen Justiz bereits vor zehn Jahren beschrieben.59 Tatsächlich ist auf der einen Seite ein unübersehbarer Trend zur befugnismäßigen Aufrüstung - gerade auch im Bereich der informationellen Zusammenarbeit der Sicherheitsbehörden ${ }^{60}-$ aus- $^{-}$ zumachen, während anderswo »Entsicherungs- bzw. Verschlankungsprozesse« - die Reformen im Sozialrecht sind nur besonders prominente Ausprägungen zu beobachten sind. ${ }^{61}$ Für das hier interessierende Phänomen ist tatsächlich von einem Paradoxon zu sprechen: »Ist einerseits, was die Bekämpfung von organisierter Kriminalität und Terrorismus betrifft, eine massive Ausweitung polizeilicher Mittel und Maßnahmen zu beobachten, versucht sich andererseits der Staat seiner Sicherheitsverantwortung in den Bereichen der traditionellen Gefahrenabwehr teilweise zu entledigen und privaten Akteuren zu überantworten ${ }^{62}$ Tatsächlich wird man schon heute konstatieren müssen, dass das Maß an öffentlicher Sicherheit, die letztlich dem Individuum nützt, bereits zu einem erheblichen Anteil an finanzielle Handlungsmöglichkeiten Privater gekoppelt ist. ${ }^{63}$ Die Ursachen hierfür liegen nicht zuletzt in einer gesetzgeberischen Untätigkeit, die der Verschiebung zugunsten privater Sicherheitsproduktion erheblichen Vorschub geleistet hat. Das staatliche Gewaltmonopol wird auf diese Weise grundsätzlich in Frage gestellt ${ }^{64}$ - ein »Luxus«, den sich ein auf Glaubwürdigkeit angewiesener Rechtsstaat nicht länger leisten sollte.

57 Vgl. dazu Thilo Weichert, Regelung zur Videoüberwachung verbesserungswürdig, Datenschutznachrichten 3/200I, 5-8, 6; ähnlich auch schon vor der Verabschiedung der Novelle Pitschas (Fn. 38), I 8 I3.

58 Amtsgericht Berlin-Mitte, NJW-RR I9 (2004), 53 I-534.

59 Martin Kutscha, Starker Staat mit Januskopf, Kritische Justiz 3 I (1998), 399-405.

60 Ein herausstehendes Beispiel stellt das Gemeinsame-Dateien-Gesetz vom I. I 2.2006 (BGBl. I 2006, 3409) dar; krit. hierzu Fredrik Roggan/Nils Bergemann, Die neue Sicherheitsarchitektur der Bundesrepublik, NJW 60 (2007), 876-88 I.

6I Krit. auch Kutscha (Fn. 5), 20.

62 Dirk Heckmann, Sicherheitsarchitektur im bedrohten Rechtsstaat, in: Ulrich Blaschke/Achim Förster/ Stephanie Lumpp/Judith Schmidt (Hrsg.), Sicherheit statt Freiheit?, Berlin 2006, 9-28, I6.

63 Ebenso Kutscha (Fn. 2), 90.

$64 \mathrm{Vgl}$. dazu auch Schenke (Fn. 2), $28 \mathrm{I}$. 